\title{
Editorial
}

\section{Pediatric Tuberculosis: All in the Family?}

\author{
Kashef Ijaz, MD, MPH; Kenneth G. Castro, MD
}

In the United States, the remarkable increase in tuberculosis (TB) (54.5\%) among young adults 25 to 44 years of age (the prime childbearing and child rearing years) from 1985 to 1992 was paralleled by marked increases in TB among children. ${ }^{1}$ During the same period, cases of TB among individuals 14 years of age and younger increased $34.1 \%{ }^{2}$ Increased rates of TB among adults may be from primary infection or disease progression from remote, latent TB infection, but increased rates among children are generally caused by recent transmission of $\mathrm{TB}$, thus representing a primary infection in the child. The occurrence of a recent TB infection in a child should be considered a sentinel event indicating transmission of Mycobacterium tuberculosis from an infectious adult or adolescent to that child and should routinely lead to the investigation of contacts to identify the potential source case or cases as well as other recently infected individuals.

Transmission of $M$. tuberculosis in healthcare facilities has been documented..$^{3-11}$ The magnitude of such institutional transmission varies considerably by the type of healthcare facility, the prevalence of TB in the community, the patient population served, the healthcare workers' occupational group, the area of the healthcare facility in which the healthcare worker works, and the effectiveness of TB infection control measures. ${ }^{12}$ The risk of transmission may be higher in areas where patients with TB are provided care before diagnosis and initiation of TB treatment and isolation precautions or where diagnostic or treatment procedures that stimulate cough are performed.

Children usually acquire TB from adults or adolescents. Children younger than 10 years are usually not contagious. ${ }^{13,14} \mathrm{~TB}$ in young children is rarely infectious, because young children are less likely than adults to have a productive cough, to generate the force needed to aerosolize organisms into droplet nuclei, or to have large numbers of infectious organisms in their sputum. There are case reports of children being implicated as the source of TB transmission to their contacts, however, some of whom developed active TB. ${ }^{15}$ Therefore, children with evidence of clinical TB, either signs or symptoms or abnormal results on chest radiographs, should be appropriately isolated until they have three serial acid-fast bacillus smears that are negative, receive appropriate treatment, and have decreased cough. ${ }^{12,14}$ Nosocomial TB infections in children's wards or children's hospitals have frequently originated from a healthcare worker or an adult visitor with unrecognized TB. ${ }^{16}$ Recommendations for healthcare facilities issued by the Centers for Disease Control and Prevention in 1994 highlight the need for instituting a hierarchy of control measures consisting of administrative, engineering, and personal protection procedures to decrease the risk of nosocomial transmission of TB. ${ }^{12}$

In this issue, Muñoz et al. ${ }^{17}$ describe the importance of targeted TB screening of accompanying adults and adult visitors of children hospitalized for suspected TB at a children's hospital in detecting unrecognized pulmonary TB, which may constitute a threat of infection to hospital staff and other patients. They emphasize that infection control procedures in pediatric facilities should take into consideration that TB in a child is a marker of recent transmission of $M$. tuberculosis from a close contact. Children, particularly during the first 5 years of life, may develop TB after a relatively short incubation period following infection with $M$. tuberculosis from a source that might not be apparent or previously identified. ${ }^{18,19}$ As suggested by Muñoz et al., this source is often in the child's household. The emphasis of screening procedures should therefore be on the adults accompanying children hospitalized with suspected TB.

The authors are from the Division of Tuberculosis Elimination, National Center for HIV, STD and TB Prevention, Centers for Disease Control and Prevention, Atlanta, Georgia.

Address reprint requests to Kashef Ijaz, MD, Division of TB Elimination, Centers for Disease Control and Prevention, 1600 Clifton Road, Mailstop E-10, Atlanta, GA 30333 . 
This is a well-conducted study at the Texas Children's Hospital. The study methodology and intervention are well described. From 1992 to 1998 , adults accompanying 59 consecutive children admitted to Texas Children's Hospital for suspected TB were prospectively evaluated to determine the frequency of undiagnosed potentially infectious disease. Annual rates of tuberculin skin test conversion in hospital employees were also obtained for the same period. Children were placed in respiratory isolation only if they or an accompanying adult had characteristics of potentially infectious disease.

However, the confinement of potentially infectious adults to the room of the child with whom they were staying raises practical considerations about the enforcement of such a policy. Such considerations include the ability to monitor the movement of potentially infectious visitors in and out of the hospital, and, more specifically, access to and from the isolation room. During the course of this study, a brief medical history was obtained from all accompanying adults. It will be important to coordinate the obtainment of this brief history with more extensive risk factor interviews likely conducted by Health Department staff during TB contact investigations. Relevant information is likely to include history of injected drug use, human immunodeficiency virus infection, alcohol use, or residence at a correctional facility, homeless shelter, or long-term-care facility.

Notably, the authors also collected and analyzed the results of annual tuberculin skin testing of hospital employees and correlated the tuberculin skin test conversions among them according to their unit or department and patient contact. However, these conversions were described as a cluster of cases instead of as a cluster of tuberculin skin test conversions. A description such as this can be misleading, as most recent tuberculin skin test conversions are likely asymptomatic and non-infectious. Individuals with TB are most often symptomatic and require both treatment and investigation of their contacts.

The study revealed that of the 105 adults screened for 59 consecutive children, $16(15 \%)$ had previously undetected pulmonary TB. This represents a TB case rate of 15,000 per 100,000, making this process an important casedetection tool. Contagious adults were associated with 14 (24\%) of the 59 children. Only 8 (13.5\%) of the 59 children required isolation. In all instances in which the adult was the patient's parent, he or she was identified as the source of infection to the child. Tuberculin skin test conversion occurred in 127 employees, for a conversion rate of 8 per 1,000 employee-years. Only 4 of these 127 employees performed activities involving direct patient contact, however. None was in contact with a family known to have a potentially contagious adult or pediatric patient.
The study appropriately concluded that infection control efforts around children with TB should focus on accompanying adults and adult visitors as a useful method to detect previously undiagnosed and potentially contagious individuals. Since the implementation of these procedures, there have been no documented cases of nosocomial $M$. tuberculosis transmission from a patient or his or her family to healthcare workers or to other patients in the authors' institution. Importantly, the authors coordinated their efforts with those of the Houston Health Department.

Other healthcare centers receiving and diagnosing $\mathrm{TB}$ in children should consider evaluating the implementation of similar procedures in an effort to improve patient outcomes, identify others in need of therapy, and prevent further transmission of $M$. tuberculosis. Such measures will prove crucial to the eventual elimination of $\mathrm{TB}$ in the United States.

\section{REFERENCES}

1. Cantwell MF, Snider DE, Cauthen GM, Onorato IM. Epidemiology of tuberculosis in the United States, 1985 through 1992. JAMA 1994;272:535-539.

2. American Thoracic Society, Medical Section of the American Lung Association. Control of tuberculosis in the United States. Am Rev Respir Dis 1992;146:1623-1633.

3. Barrett-Connor E. The epidemiology of TB in physicians. JAMA 1979;241:33-38.

4. Brennen C, Muder RR, Muraca PW. Occult endemic TB in a chronic care facility. Infect Control Hosp Epidemiol 1988;9:548-552.

5. Goldman KP. TB in hospital doctors. Tubercle 1988;69:237-240.

6. Catanzaro A. Nosocomial TB. Am Rev Respir Dis 1982;125:559-562.

7. Ehrenkranz NJ, Kicklighter JL. TB outbreak in a general hospital: evidence of airborne spread of infection. Ann Intern Med 1972;77:377-382.

8. Haley CE, McDonald RC, Rossi L, Jones WD Jr, Haley RW, Luby JP. TB epidemic among hospital personnel. Infect Control Hosp Epidemiol 1989;10:204-210.

9. Hutton MD, Stead WW, Cauthen GM, Bloch AB, Ewing WM. Nosocomial transmission of TB associated with a draining tuberculous abscess. I Infect Dis 1990;161:286-295.

10. Kantor HS, Poblete R, Pusateri SL. Nosocomial transmission of TB from unsuspected disease. Am J Med 1988:84:833-838.

11. Lundgren R, Norrman E, Asberg I. Tuberculous infection transmitted at autopsy. Tubercle 1987;68:147-150.

12. Centers for Disease Control and Prevention. Guidelines for preventing the transmission of Mycobacterium tuberculosis in health-care facilities, 1994. MMWR 1994;43(RR-13):1-132.

13. Wallgren A. On the contagiousness of childhood tuberculosis. Acta Paediatr 1937;22:229-234.

14. Lawrence RM. Tuberculosis in children. In: Rom WN, Garay SM, eds. Tuberculosis. Boston: Little, Brown; 1996:675-688.

15. Curtis AB, Ridzon R, Vogel $R$, et al. Extensive transmission of Mycobacterium tuberculosis from a child. N Engl J Med 1999;341:14911495.

16. Starke JR. Transmission of Mycobacterium tuberculosis to and from children and adolescents. Semin Pediatr Infect Dis 2001;12:115-123.

17. Muñoz FM, Ong LT, Seavy D, Medina D, Correa A, Starke JR. Tuberculosis among adult visitors of children with suspected tuberculosis and employees at a children's hospital. Infect Control Hosp Epidemiol 2002,23:568-527.

18. Brailey, M. Mortality in tuberculosis positive infants. Milbank $Q$ 1937;15:36.

19. Lobato MN, Mohle-Boetani JC, Royce SE. Missed opportunities for preventing tuberculosis among children younger than five years of age. Pediatrics 2000;106:E75. 